\title{
Unusual Case of New Onset Diabetes Mellitus Presenting with Diabetic Ketoacidosis and Cerebral Edema with Literature Review
}

Nitasa Sahu*, Emma Punni, Chandra Chandran and Medhat Ismail

Department of Internal Medicine, St. Joseph's Regional Medical Center, New York Medical College, Paterson, USA

*Corresponding author: Nitasa Sahu, Department of Internal Medicine, St. Joseph's Regional Medical Center, New York Medical College, 703 Main Street, Paterson, NJ 07503, USA, Tel: 973-754-2431; E-mail: nitasasahu@gmail.com

Received date: October 24, 2016; Accepted date: October 30, 2016; Published date: October 31, 2016

Copyright: @ 2016 Sahu N, et al. This is an open-access article distributed under the terms of the Creative Commons Attribution License; which permits unrestricted use; distribution; and reproduction in any medium; provided the original author and source are credited.

\begin{abstract}
Diabetic ketoacidosis (DKA) is typically treated with volume replacement (most commonly normal saline), insulin and monitored via serial chemistry and glucose lab values. Cerebral edema, a complication occurring in approximately $1 \%$ of DKA presentations in children, with a mortality of $40-90 \%$, has no clear identifiable risk factors. While many cases have been reported in children, there are only a few cases of clinically significant cerebral edema in adults. It is postulated the underlying mechanism is similar to that in children; excessive fluid resuscitation, rapid reduction in plasma osmolarity, and/or the administration of sodium bicarbonate. We are reporting a case of a 26 year old male with no prior medical history, who presented in diabetic ketoacidosis and was treated as per the American Diabetic Association guidelines, however, deteriorated rapidly after acute complaints of headache and irritability consistent with diffuse cerebral edema.
\end{abstract}

Keywords: Diabetic ketoacidosis; Cerebral edema; Adult; Mortality

\section{Introduction}

Diabetic ketoacidosis typically occur in Type 1 diabetic patients, however, can be seen in Type 2 diabetics that neglect their insulin or have a precipitating factor, such as an underlying infection or other type of stressor on the body requiring more insulin [1]. Complications do occur in DKA, most commonly hypoglycemia, hypophosphatemia, hypokalemia, etc. Cerebral edema is a rare, but severe complication in adults and the mechanism behind it is controversial and not proven.

Here, we present a case of a 26 year old African American male who died within 36 hours of presentation of DKA causing cerebral edema. A brain autopsy performed revealed diffuse moderate brain edema and acute extensive bilateral cortical necrosis [2].

\section{Case Report}

A 26 year old African American male with no prior medical history presented to the Emergency Department (ED) for complaints of progressive lethargy, nausea, vomiting and abdominal pain. The patient reported visiting the Caribbean Island for ten days and returned one week prior to presenting to the ED. Prior to leaving for Jamaica, he denied any of the aforementioned symptoms. During his stay, he noticed he was drinking excessive water, urinating more often, and felt very lethargic. At that time, however, the patient maintained good appetite and denied any nausea or vomiting. Upon returning, he experienced vomiting in addition to his other symptoms and had gone to an urgent care center to have labs drawn. Before he could obtain the results, he presented to the ED due to new onset right lower quadrant abdominal pain, more frequent vomiting, and extreme fatigue. Vitals signs taken showed a temperature of $96.4^{\circ} \mathrm{F}$, heart rate of $119 \mathrm{bpm}$, respiratory rate of 18 , and blood pressure of $147 / 83 \mathrm{mmHg}$ [3]. Physical exam revealed an extremely dry oral mucosa and dry skin; otherwise all other organ systems were unremarkable.
Initial laboratory values revealed a WBC of $22.2 \times 10^{3} / \mathrm{L}$, hemoglobin of $17.5 \mathrm{mg} / \mathrm{dL}$, hematocrit of 54.3 , platelet count of 198 , sodium of $132 \mathrm{meQ} / \mathrm{L}$ (corrected sodium $144 \mathrm{meQ} / \mathrm{L}$ ) [4], potassium of $6.4 \mathrm{meQ} / \mathrm{L}$, chloride of $92, \mathrm{CO}_{2}$ of 7 , blood urea nitrogen of 42 , creatinine of 2.0 and glucose of 838. Serum acetone was large and arterial blood gas revealed a $\mathrm{pH}$ of $7.14, \mathrm{CO}_{2}$ of $17, \mathrm{O}_{2}$ of 114 , and $\mathrm{HCO}_{3}$ of 5.8 on $21 \% \mathrm{FiO}_{2}$. Lactic acid was 4.4. Based on these findings, the patient was admitted to the Medical Intensive Care Unit for diabetic ketoacidosis, SIRS, and acute kidney injury. His DKA was treated as per ADA guidelines, and had received 2 litres of $0.9 \%$ normal saline bolus and 10 units of subcutaneous insulin bolus after which patient was started on an insulin drip. One and a half hours after being started on the insulin drip, the patient complained of an occipital headache and was given Tylenol $975 \mathrm{mg}$ PO with no relief. As per the mother, he was becoming more irritable with no resolution of the headache and was then given morphine. Moments after, the patient was difficult to arouse and was found to have fixed and dilated pupils of $6 \mathrm{~mm}$. He underwent a stat head CT which were consistent with findings of severe diffuse brain swelling (Figure 1a and 1b). An ICP bolt monitor was placed, and an ICP was measured to be $35-38 \mathrm{mmHg}$. The patient was given mannitol and started on hypertonic saline immediately. Even after the ICP improved to $<20 \mathrm{mmHg}$, there was no noted change in his neurological exam. He remained unresponsive with loss of pupillary, corneal, gag, and cough reflex. In less than 48 hours after presentation, the patient developed Pulseless Electrical Activity, for which cardiac resuscitation was initiated. The patient then went into Ventricular Tachycardia for which he was shocked and had return of spontaneous circulation, however, then lost pulse again without a successful outcome. An autopsy of the brain performed showed moderate brain edema and acute extensive bilateral necrosis with fragmentation of several structures with perivascular and parenchymal micro-haemorrhages. All blood cultures and urine cultures were negative. 


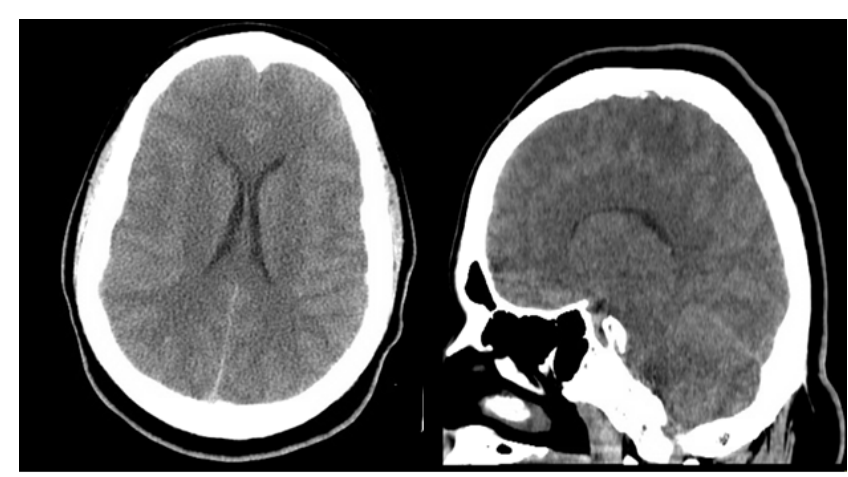

(a)

(b)

Figure 1: $(\mathrm{a}$ and $\mathrm{b})$ : Severe brain swelling with obliteration of the sulci, ventricles and perimesencephalic cisterns.

\section{Discussion}

Cerebral edema in adults is a rare, however, fatal complication of diabetic ketoacidosis (DKA). It has been studied in the pediatric population and even then only affects $1 \%$ of patients with DKA. There are very few cases that have been reported in the adult population to the best of our knowledge [5]. Although most cases of cerebral edema occur within 4-12 hours of initiating treatment, it can occur at any point within 12 hours of starting treatment [6]. Signs and symptoms typically include headache, vomiting, lethargy, and change in mental status. Often, these patients will deteriorate very rapidly and without any warning. Delayed diagnosis and treatment thus contribute to the high mortality rate of $70-90 \%$ [7]. Several mechanisms have been theorized such as a rapid decline in plasma osmolality, the administration of bicarbonate, a high fluid resuscitation rate, the use of hypotonic fluids, and an increased rate of decline in blood glucose level [7]. In the table provided (Table 1), one can see the laboratory values coinciding with time, thus negating these theories. The few case reports identified are discussed below with a literature review.

\begin{tabular}{|l|l|l|l|l|l|l|}
\hline & $\begin{array}{l}\text { Sodium } \\
\text { (Meq/L) }\end{array}$ & $\begin{array}{l}\text { Potassiu } \\
\mathbf{m} \text { (Meq/L) }\end{array}$ & $\begin{array}{l}\text { Chloride } \\
\text { (Meq/L) }\end{array}$ & $\begin{array}{l}\text { CO2 } \\
\text { (Meq/L) }\end{array}$ & $\begin{array}{l}\text { Glucose } \\
\text { (Mg/dL) }\end{array}$ & $\begin{array}{l}\text { Serum } \\
\text { Osmolality } \\
\text { (mOsm/kg) }\end{array}$ \\
\hline Time 0 & 132 & 6.4 & 92 & 7 & 838 & 326 \\
\hline $\begin{array}{l}+\quad 6 \\
\text { hours }\end{array}$ & 139 & 3.8 & 100 & 8 & 471 & 315 \\
\hline $\begin{array}{l}+\quad 10 \\
\text { hours }\end{array}$ & 139 & 3.9 & 103 & 11 & 486 & 314 \\
\hline $\begin{array}{l}+\quad 12 \\
\text { hours }\end{array}$ & 142 & 4.8 & 113 & 10 & 440 & 317 \\
\hline $\begin{array}{l}+\quad 16 \\
\text { hours }\end{array}$ & 149 & 5.7 & 121 & 12 & 354 & 326 \\
\hline $\begin{array}{l}+\quad 20 \\
\text { hours }\end{array}$ & 157 & 5.3 & 128 & 15 & 366 & 341 \\
\hline
\end{tabular}

Table 1: Laboratory values coinciding with time.

In 2010, Haringhuizen et al. [8] reported a similar case of a young male without prior medical history who presented with his first episode of DKA. He reported that despite being treated as per guidelines, the patient suffered a fatal case of cerebral edema. Two potential mechanisms may have played a role in this case after analyzing the laboratory values. The first being a rapid fall in glucose was noted, however, was also accompanied by an increasing plasma sodium level thus allowing only a small change in plasma osmolality. A second mechanism was theorized after post-mortem studies revealed pulmonary edema. In such a case, Haringhuizen postulated that the pulmonary edema was causing persistent respiratory acidosis and that increased $\mathrm{pCO}_{2}$ levels would have allowed intracellular acidosis to mimic the same pathophysiology as administering bicarbonate which, however, was not the case in our patient [8].

Similarly, another case was reported by Troy et al. [9] in 2005, of a 27 year old male with no known history of diabetes who went on to develop cerebral edema after presenting with a first episode of DKA and deteriorated very rapidly. The patient initially presented with progressive polyuria, polydipsia, along with intermittent global headaches and mild photophobia. On arrival to the Emergency Department, the patient also experienced one episode of generalized seizure. A lumbar puncture demonstrated an elevated opening pressure of 55 centimeters $\mathrm{H}_{2} \mathrm{O}$. Given the fact that the patient had presented with an elevated opening pressure during DKA and prior to initiating treatment, the authors propose that the rapidly lowering serum osmolality may not be the sole cause of cerebral edema.

A third case report published in the American Journal of Emergency Medicine in 2005 described a 31 year-old male with no prior medical history who presented to the ED in DKA. After following the protocol, within 45 minutes, the patient's mental status had deteriorated prompting a CT scan which showed diffuse cerebral edema. The patient also had pancreatitis and was treated accordingly. This case, however, differs from the previous two and our case in that the patient made a full recovery with no neurologic deficits after seven days of hospitalization. The fact that this case report appears to be the only one with a positive outcome, a study by Azzopardi suggested a possible predisposition to developing cerebral edema [10]. His study concluded there was more brain tissue density between CT scans of patient's in DKA compared with those CT scans taken more than six months after an episode of DKA, which posed no difference in those that were in the control group [11]. Based on these findings, there is possibility that the degree of dehydration predisposes one to cerebral edema.

Of interest, there were findings of perivascular and parenchymal microhemorrhages on microscopy in our patient. According to Viswanathan, microhemorrhages are most commonly associated with older age, high blood pressure, smoking, and a history of ischemic stroke, however, have been seen in healthy adults as well as cerebral amyloid angiopathy [12]. Because this patient had none of the above risk factors, another etiology may have been from the hypernatremia that was induced after being initiated on hypertonic saline. In 1979, authors Young and Traux reported the case of a 12 year old adolescent male with a history of diabetes who was given $500 \mathrm{ml}$ of hypertonic infusion after which he went on to develop hypernatremia followed by seizures, coma and eventually death [13]. A CT head showed numerous small subcortical hemorrhages which were confirmed postmortem [13]. The underlying mechanism of such micro hemorrhages in the setting of hypernatremia may likely be due to stretching and tearing of vessels in the setting of the stress created by shrinkage of brain tissue within the rigid cranium. We believe that a 
Citation: Sahu N, Punni E, Chandran C, Ismail M (2016) Unusual Case of New Onset Diabetes Mellitus Presenting with Diabetic Ketoacidosis and Cerebral Edema with Literature Review. J Nephrol Ther 6: 262. doi:10.4172/2161-0959.1000262

Page 3 of 3

similar mechanism played a role in causing the findings of micro hemorrhages for our patient.

At this time, the exact pathophysiology behind the development of cerebral edema remains poorly understood and no literature is able to clearly support any particular theory. In 1973, Arieff and Kleeman conducted a clinical study on rabbits to investigate the pathophysiology of cerebral edema during the treatment of DKA [14]. Their theory was that during hyperglycemia, the brain generates 'ideogenic osmoles' as a mechanism to prevent brain cells from shrinking as a result of an increase in extracellular osmolality that occurs during DKA. These 'ideogenic osmoles' help create an equilibrium between the intracellular brain cells and the extracellular environment. However, rapid drop in blood glucose level via insulin administration can cause the extracellular osmolality to drop suddenly, causing an osmotic shift of water into the now hypertonic brain cells, thus causing cerebral edema [15]. The hypothesis of ideogenic osmoles', however, has never been proven. Although other mechanisms have been suggested, such as rapid administration of fluids, a rapid fall in serum osmolality and use of bicarbonate, these are only postulations with nothing proven. All aforementioned case reports did not identify with any of these possible mechanisms, thereby invalidating them.

\section{Conclusions}

In conclusion, although cerebral edema is a fatal complication of DKA that primarily affects children, we recommend physicians to hold a high index of suspicion in young adults with new onset DKA who, after being started on treatment, show any change in their neurological status, such as worsening headache and/or altered mental status. Early imaging, diagnosis and prompt intervention may help in lowering the mortality rate.

\section{Conflict of Interest}

Authors declare that there is no conflict of interest regarding the publication of this manuscript.

\section{References}

1. Glaser N, Barnett P, Mccaslin I, Nelson D, Trainor J, et al. (2001) Risk Factors for Cerebral Edema in Children with Diabetic Ketoacidosis. New England Journal of Medicine 344: 264-269.

2. Hardern R, Quinn N (2003) Emergency management of diabetic ketoacidosis in adults. Emergency Medicine Journal 20: 210-213.

3. Trachtenbarg D (2005) Diabetic Ketoacidosis. Am Fam Physician 71: 1705-1714.

4. Katz MA (1973) Hyperglycemia-induced hyponatremia--calculation of expected serum sodium depression. N Engl J Med 289: 843-844.

5. Hiller KM, Wolf SJ (2005) Cerebral edema in an adult patient with diabetic ketoacidosis. American Journal of Emergency Medicine 23: 399-400.

6. Azzopardi J, Gatt A, Zammit A, Alberti G (2002) Lack of evidence of cerebral oedema in adults treated for diabetic ketoacidosis with fluids of different tonicity. Diabetes Res Clin Practice 57: 87-92.

7. Katsilambros, Nicholas (2011) Diabetic Emergencies: Diagnosis and Clinical Management. Chichester, West Sussex, UK: Wiley-Blackwell.

8. Haringhuizen A, Tjan DH, Grool A, van Vugt R, van Zante AR (2010) Fatal cerebral oedema in adult diabetic ketoacidosis. Neth J Med 68: 35-37.

9. Troy PJ, Clark RP, Kakarala SG, Burns J, Silverman IE, et al. (2005) Cerebral edema during treatment of diabetic ketoacidosis in an adult with new onset diabetes. Neurocrit Care 2: 55-58.

10. American Diabetes Association (2004) Hyperglycemic Crises in Diabetes. Diabetes Care 27: s94-s102.

11. Viswanathan A, Chabriat H (2006) Cerebral Microhemorrhages. Stroke 37: 550-555.

12. Young RSK, Truax BT (1979) Hypernatremic hemorrhagic encephalopathy. Annals of Neurology 5: 588-591.

13. Luttrell CN, Finberg L (1959) Hemorrhagic encephalopathy induced by Hypernatremmia. AMA Arch Neurol Psychiatry 81: 424-432.

14. Arieff AI (1989) Cerebral edema complicating nonketotic hyperosmolar coma. Miner Electrolyte Metab 12: 383-389.

15. Arieff Al, Kleeman CR (1973) Studies on mechanisms of cerebral edema in diabetic comas. Effects of hyperglycemia and rapid lowering of plasma glucose in normal rabbits. J Clin Invest 52: 571-583. 